\title{
TipQAD: an automated tool for quantifying apical fluorescence dynamics in tip-growing cells
}

\author{
Asongu L. Tambo1, Bir Bhanu1 ${ }^{*}$, Nan Luo ${ }^{2}$, Duoyan Rong ${ }^{2}$, Fei Wang², Christian Craddock², \\ Irene Lavagi ${ }^{2}$, and Zhenbiao Yang ${ }^{2 *}$ \\ ${ }^{1}$ Department of Electrical and Computer Engineering, Center for Research in Intelligent Systems, \\ University of California at Riverside, Riverside, CA 92521, USA \\ ${ }^{2}$ Center for Plant Cell Biology, Institute of Integrative Genome Biology, Department of Botany and \\ Plant Sciences, University of California at Riverside, Riverside, CA 92521, USA \\ *bhanu@cris.ucr.edu, zhenbiao.yang@ucr.edu
}

\section{ABSTRACT}

Cell polarity is a fundamental property essential for the function and development of all cellular organisms. Tip growth is an extreme form of polar growth requiring spatiotemporally dynamic but highly coordinated cellular activities. Quantification of these dynamic activities is important for the systematic study of the mechanisms controlling cell polarity, but an automated and unbiased method for analyzing and quantification of tip-growing cells has been missing. In this paper, we developed a computational model and an associated tool called TipQAD for quantifying the spatiotemporal dynamics of fluorescence protein (FP) labeled molecules or structures in tipgrowing cells. This tool robustly and accurately measured the spatial distribution and temporal dynamics of FP-labeled proteins in the cytoplasm or the plasma membrane at the tip region of several types of tip-growing cells. We also demonstrated its ability to analyze data from FRAP experiments. In conclusion, this tool showed great potential for automated measurements of the spatiotemporal dynamics of fluorescent-labeled cellular molecules and structures.

\section{Introduction}

Cell polarity describes the asymmetric property of a cell in all cellular organisms and is critical for cell function and development. It is required for cell movement, morphogenesis, 
differentiation, polar and directional growth, and so on. All forms of polarity are achieved through the spatiotemporal dynamic networks of regulatory molecules and cellular structures. As such, there is a need for automated tools that can quickly and accurately quantify the dynamics of these cellular molecules and structures in time and space. Tip growth, also known as apical cell growth, is an extreme form of polar growth characterized by a unidirectional expansion from a particular polarized region of the cell and is frequently observed in many cell types such as pollen tubes, root hairs ${ }^{1}$, fungal hyphae, and animal neurons. The networks of signal or structural molecules for regulating the polarity and building the cell are highly dynamic and coordinated during the polar growth, as the nascent cell membrane and cell wall materials are inserted into the same region where the networks are localized. Hence tip-growing cells provide an excellent system for the study of spatiotemporally dynamic cellular activities.

Among model systems for studying the molecular/cellular network underlying tip growth, pollen tube is one of the most studied because it can be conveniently manipulated and imaged in vitro and because of various genetic and molecular tools available for this system. As in other walled tip-growing cell types, the growth of pollen tubes at the apex requires the local expansion of the cell wall driven by turgor pressure, while the mechanical properties of the cell wall determine the morphology of the cell ${ }^{2-5}$. The precise control of the deposition of cell surface materials is regulated by a highly coordinated signaling network, involving vesicular trafficking, cytoskeletal dynamic/re-organization, ROP (Rho-like GTPase from plants) signaling and fluxes of ions such as calcium ${ }^{3,6,7}$. This network is characterized by its remarkable spatiotemporal dynamics. Spatially, it is highly polarized: dense populations of exocytic vesicles, supported by longitudinal actin cables and an apical actin network, move towards the extreme apex of pollen tubes in a "reverse fountain" pattern, delivering cell membrane and cell wall components to the tip $^{7,8}$, while endocytosis retrieves excessive materials from the subapical or apical regions ${ }^{9}$. Active ROP1 and its signaling network, localized to the cell tip, has been shown to play an essential role in maintaining the cell polarity by regulating actin filaments and exocytosis ${ }^{10-12}$. Calcium ions also show a tip-high gradient and are crucial for pollen tube growth by participating in multiple pathways including the ROP signaling ${ }^{13}$. Temporally, this system is oscillatory and extremely dynamic: ROP1 activity, calcium concentration, and accumulation of vesicles at the cell tip exhibit rapid and correlated oscillations resulting in oscillating growth ${ }^{14-16}$. The mechanisms underlying the polar growth of other tip-growing cells, such as root hair, fungal 
hyphae, and neurons, are evolutionarily conserved. Therefore, the study of polar growth in these cell models relies heavily upon the analysis of the distribution and dynamics of various molecules and structures, yielding large amounts of video image data that require quantification and interpretation, which is extremely time-consuming by manual measurements.

In the current study, we develop TipQAD (Tip Quantification of Apical Dynamics), which is an automated tool for analyzing the spatial distribution and temporal dynamics of fluorescently labeled molecules/structures on the plasma membrane (PM) and in the cytoplasm at the apex of tipgrowing cells. The proposed method is novel for the following reasons: (1) tip detection is done by optimizing intuitive functions based on the expectations of the geometry of tip-growing cells ${ }^{4,6,17}$; (2) a fast, fully automated measurement method is developed to replace the manual measurements that are currently made in the study of tip growth. Given a video frame, the method performs image segmentation (using user feedback on the first image) to obtain the shape of the cell. Then tip detection is used to determine the location and vertex of the tip region. Based on user-defined distances, measurements are made along the cell membrane and in the cytoplasm if required. Here we used this tool to analyze the spatiotemporal dynamics of fluorescence protein (FP)-tagged proteins localized in the cytoplasm in Arabidopsis pollen tubes, Arabidopsis root hairs. The accuracy of our automated program was confirmed by comparing the results with manual measurements.

\section{Results}

\section{Computer vision techniques mediated recognition of tip-growing cells}

Our computational model assumes that a tip-growing cell is made up of a cylindrical base and a hemispherical tip ${ }^{4,6,17}$ (Figure 1). After segregating the tip-growing cell, our model focuses on determining the best location for the apex (vertex) of the tip-growing cell in a given image(s). To ensure continuity in detecting the cell apical region, we assume that the apical region of the cell in the current frame is close to that of the previous frame. As such, we can limit the search space for the apical region in the current frame to the contour locations that are next to the apical region in the previous frame. Finally, our method assumes that the frames to be analyzed contain a single pollen tube. 


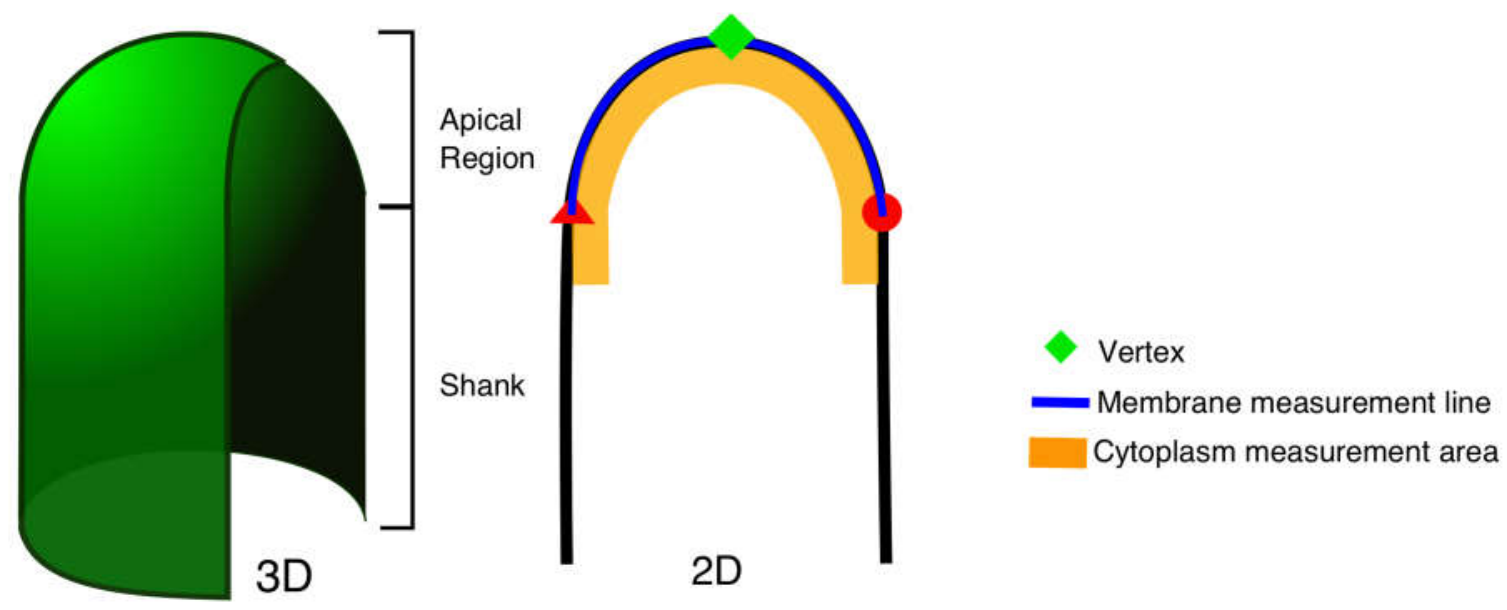

Figure 1: The schematic diagram of the apical region and shank of the pollen tube in 3D with an accompanying 2D diagram. The $2 \mathrm{D}$ diagram shows the measurement regions: membrane (blue) and tip cytoplasm(orange)

\section{Segmentation of the tip-growing cell from fluorescent images}

The input images for our computational model have to be fluorescence images that show the localization and intensity of an FP-tagged protein inside the cell. Image segmentation treats fluorescence images as an intensity map. After smoothing the image with a Gaussian filter, a Laplacian is used to detect regions of varying pixel intensities (regions with edges). This is done using a LoG-filter that takes the form:

$$
\operatorname{LoG}(i, j))=\frac{1}{\pi \sigma^{4}}\left[\frac{i^{2}+j^{2}}{2 \sigma^{2}}-1\right] e^{-\frac{i^{2}+j^{2}}{2 \sigma^{2}}}
$$

where $i$ and $j$ are the row and column positions of a pixel respectively, and $\sigma=\frac{R_{L o G}}{3}$ is the standard deviation of the Gaussian filter. An entropy filter (of radius $R_{\text {Entropy }}$ ) is used to compute the entropy of the image. In this case, entropy is defined as the measure of contrast in a given region: regions with lower variation in pixel intensities have lower entropy as compared to regions with a larger pixel intensity variation. Otsu's thresholding algorithm is used on the result of the entropy calculation to obtain a segmentation of the image. The initial values for $R_{L O G}$ and $R_{\text {Entropy }}$ are userdefined. At the start of the program, the user is allowed to change these values to ensure accurate segmentation of the cell region.

While this is a very effective segmentation procedure, it creates some false positive regions. Simple morphological operations (erosion (radius $=0.7 r_{\text {ent }}$ ) followed by hole-filling) are used 
to obtain simply connected regions without holes. Regions with sizes of less than 10 pixels are discarded. Of the remaining regions, the region with the highest average intensity is designated as the cell region. The result of this process is a binary image that shows a single cell region as the foreground (pixel value $=1$ ). This binary image is smoothed to promote contour smoothness. This technique has been used in our previous work segmentation. Shape smoothing is the final phase of the segmentation process, which is done to promote a smooth contour of the cell shape. A smooth contour is helpful in determining the curvature along the contour of the cell. The smoothing process is an iterative process performed on the binary segmentation, and it terminates when there is no change in the shape of the cell between consecutive iterations or the maximum number of iterations (500) is reached. Let the result of segmentation be the image $I$ of size $r \times c$ (r-rows and ccolumns). At each iteration, a temporary image $I_{\text {temp }}^{i}$ (temporary Image at iteration $i$ ) is created as:

$$
I_{\text {temp }}^{i}=I^{i-1}+I_{E}+I_{D}
$$

where $I^{i-1}$ is the result of the previous iteration, $I_{D}$ is formed by adding a layer of pixels to the border of the object in $I^{i-1}$ and $I_{E}$ is formed by removing one layer of pixels at the border of the object in $I^{i-1}$. $I_{\text {temp }}^{i}$ has values between [0 3]. This temporary image is filtered using:

$$
I_{s}=\left(G_{\sigma} \otimes_{2} I_{\text {temp }}^{i}\right)_{(r \times c)}
$$

where $\bigotimes_{2}$ is the $2 \mathrm{D}$-convolution operator, $G_{\sigma}$ is a $2 \mathrm{D}$-Gaussian filter with a standard deviation of $\sigma .(\cdot)_{(r \times c)}$ enforces the condition that $\operatorname{size}\left(I_{s}\right)=\operatorname{size}\left(I_{\text {temp }}^{i}\right)$. This is achieved by zero-padding the edges of $I_{\text {temp }}^{i}$ with $3 \sigma$ rows/columns to each edge. In this version of the program, the standard deviation of the filter is set to 1.5 pixels. A new segmentation is created from the smoothed image by applying a threshold the following thresholding scheme: a pixel location (x, y) is considered stable $(I(x, y) \geq 2)$ if it belongs to two of the three sub-images in equation (1) namely $I^{t-1}, I_{E}$ and $I_{D}$. Applying this threshold creates a new binary image as:

$$
I_{\text {update }}^{i-1}(x, y)= \begin{cases}1 & I_{s}(x, y) \geq 2 \\ 0 & I_{S}(x, y)<2\end{cases}
$$

The new binary image $\left(I_{\text {update }}^{i-1}\right)$ is compared to the result of the previous iteration $\left(I^{i-1}\right)$. If there is a change in the shape, then equations (2) - (4) are repeated. If there is no change in the shape of the object, a final image is produced by dilating $I_{\text {update }}^{i-1}$ using a disk element:

$$
I^{i}=\operatorname{dilate}\left(I_{\text {update }}^{i-1}, D_{\sigma+1}\right)
$$


where $\operatorname{dilate}(\cdot)$ is the dilation function, $D$ is a disk element of radius $\sigma+1 . \sigma$ has the same definition as in (Error! Reference source not found.).

\section{Estimation of the apex of the tip-growing cell}

After segmented the tip-growing cell from the given images, we aim to identify the apex (tip vertex) of the apical growing tip. Estimation of the apex of the growing tip is modeled as an ellipse that best describes this two-dimensional region. The conic section whose equation is given by:

$$
a_{1} x^{2}+a_{2} x y+a_{3} y^{2}+a_{4} x+a_{5} y+a_{6}=\boldsymbol{G}_{\boldsymbol{i}} \boldsymbol{a}=0
$$

represents an ellipse ${ }^{18}$ if $\left(4 a_{1} a_{3}-a_{2}^{2}=1\right) \cdot \boldsymbol{a}=\left[a_{1} \ldots a_{6}\right]$ are estimated coefficients, $\boldsymbol{G}=$ $\left[x^{2}, x y, y^{2}, x, y, 1\right]$ is an $(N \times 6)$ data matrix corresponding to the $i^{\text {th }} 2 \mathrm{D}$-line segment and $\left\{\left(x_{i}, y_{i}\right), i \in 1, \ldots, N\right\}$ represents the $\mathrm{x}$ - and $\mathrm{y}$-coordinates of the points on a line segment. The center of this ellipse $\left(c_{x}, c_{y}\right)$ is obtained using:

$$
\left[\begin{array}{l}
c_{x} \\
c_{y}
\end{array}\right]=\left[\begin{array}{cc}
2 a_{1} & a_{2} \\
a_{2} & a_{3}
\end{array}\right]^{-1}\left[\begin{array}{l}
a_{4} \\
a_{5}
\end{array}\right]
$$

Given that the equation of an ellipse can be made to fit almost any continuous line segment of the cell shape, the task becomes one of finding the optimal/best location for the tip. The intuition for our solution is that overlapping line segments around the tip will have different coefficients $\left(\boldsymbol{a}_{i}\right)$, but all of them will have approximately the same ellipse center $\left(c_{x}, c_{y}\right)$. So, the tip candidates are the contour points whose ellipse center location has the most votes. In the ellipse-fitting process, the contour is split into overlapping segments of length:

$$
l=\pi r
$$

where $r$ is the radius of the cell region. This radius $(r)$ is based on the cell shape obtained from segmentation (Figure 2A(I)). A distance transform on this shape finds the minimum distance of each cell pixel to the cell contour. $r$ is the maximum value of these distances.

We use a voting scheme to search for the tip region. For each line segment $(l)$ from the cell contour, the solution to equations (6)-(7) provides the location of the ellipse center ${ }^{18,19}$. The vote at this center location is incremented by 1 . This voting process continues for all the line segments. The set of line segments, whose ellipse-center location gets the highest votes, become the candidates for the contour of the cell tip (see Figure 2 A(II) where the dotted points inside the cell indicate the number of votes received by that location as an ellipse center). The result of this process is the list of candidate tip locations. 


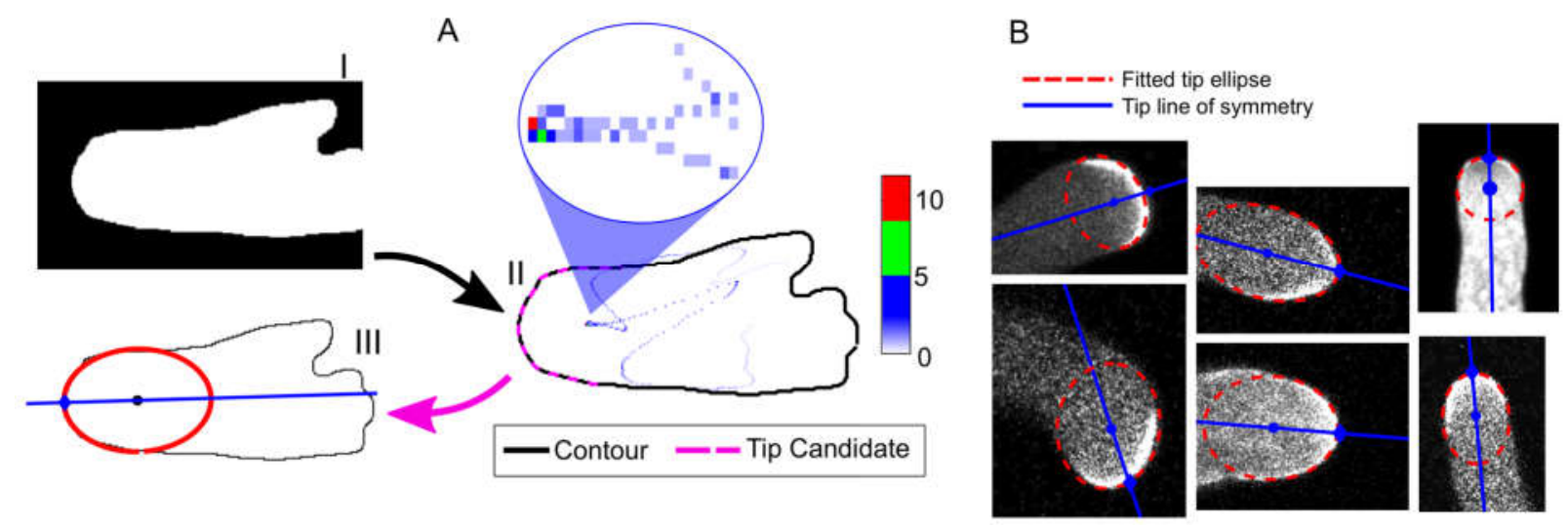

Figure 2: Tip location procedure on a sample-segmented image. (A) The tip localization method starting with the segmented image (I). Image (II) shows the cell contour, the tip candidates, and the votes received by each point as an ellipse center. A close-up of the tip region shows the center-location with the highest votes (in red). Image (III) shows an ellipse that is fitted to the tip candidates, and the vertex location. (B) Examples of tip detection on sample images with various tip widths. The solid line indicates the tip line of symmetry that is used to determine the tip vertex.

Note that in the images shown in Figure 2B, the major axis (longest radius) of the tip ellipses is not guaranteed to fall at the correct vertex of the tip region (e.g. Figure 2B, top left and bottom center). The location of the vertex is important because it denotes the geometric center of the tip region, from which point the distance arclength (a user-controlled parameter that defines half the length of membrane measurement line) is determined for membrane measurements. To find the best (optimal) vertex location, we employ the following iterative scheme. After the tip detection phase, the current tip candidates (Figure 2A-II) enclose only a small portion of the cell shape. As such, we iteratively add one pixel (one point) of the cell contour to each end of the tip candidates, thereby including more of the shank in the tip region. An ellipse is fitted to this new region and the intersection between the major axis of this ellipse and the tip candidates is noted. This process of increasing the tip region continues until the last five intersection-locations between the tip candidates and fitted ellipses are the same. Alternatively, the process can go on for fifty iterations and the average of the tip intersection, weighted by the corresponding tip area, becomes the tip vertex location.

When analyzing a video sequence, we impose the condition that the pixel locations of the tip in the current frame should be close to those in the previous frame. As such, instead of repeating the entire procedure outlined above, we focus on the points in the new curve that are within a specified distance of the tip points in the previous frame. This distance is set to $25 \%$ of 
the detected cell radius at the tip. The newly acquired tip candidate points undergo the above curvature method for vertex detection and the arclength-parameter is used to find the tip points for the current frame.

\section{Finding the optimal membrane measurement location}

The result of segmentation is the contour of the cell. This contour represents where the cell wall/membrane of the cell. However, the location of this contour may not necessarily match with that drawn by someone with knowledge of the pollen tube shape. Resolving this discrepancy is important in ensuring that the membrane measurements made by TipQAD closely resemble those obtained by manually drawing on the tip region. This resolution requires a search, from the original tip contour in the direction of the cytoplasm, for the optimal measurement location. We incorporate a winsize parameter, which determines how far into the cytoplasm the algorithm should search for the best measurement line. If winsize $=0$, then the original tip contour is used as the measurement line. Large values of winsize increase the search area for the membrane line. The value of winsize should be based on the radius of the cell: if the images have small pollen tubes, then winsize should be less than 5 .

\section{User-defined measurements of cytoplasm intensity through adjusting two parameters (Tiplength, Depth)}

The measurement region within the cytoplasm is determined by two parameters: Tiplength and depth. Tiplength parameter is the length of the region along the cell contour on either side of the vertex. This means that the width of the measurement region from end-to-end is $2 \times$ Tiplength. The depth parameter determines how far into the cytoplasm the measurement region goes. The values for these parameters can be altered from their default values to best match the desired measurement region. For a given cytoplasm region of an image, the average pixel value within the region is recorded. If depth $=0$, then the measurement region is the cell contour at the tip region with a width of $2 \times$ Tiplength. 




Figure 3: The effect of Tiplength and Depth parameters on tip cytoplasm measurements over four videos (826 images total).

(a) Sample image showing measurement regions. The first image shows the manually drawn measurement region. The remaining 4 images show automatic cytoplasm measurement regions (orange) with increasing measurement depths when Tiplength $=20 \mu \mathrm{m}$.

(b)-(e) Median of cytoplasm measurement accuracy at specific values of Tiplength for various values of depth over 4 experimental videos. Plot (e) has the most accurate values when Tiplength $=20 \mu \mathrm{m}$ and depth $=1.75 \mu \mathrm{m}$ or $2 \mu \mathrm{m}$. The measurement regions for these two depths are the last two images of (a) respectively.

Figure 3 shows the results of comparing measurements from automatic cytoplasm regions to manual measurements. The manual measurements were obtained using ImageJ software and a sample of the measurement region is shown in the first image of Figure 3(a). The rest of the images in Figure 3(a) show cytoplasm regions for Tiplength $=20 \mu \mathrm{m}$ and various values of depth. We explore different combinations of values for tiplength and depth:

$$
\begin{gathered}
\text { Tiplength }=\left[\begin{array}{llllll}
5 & 10 & 15 & 20
\end{array}\right] \mu \mathrm{m} \\
\text { depth }=\left[\begin{array}{lllllll}
0 & 0.5 & 1.0 & 1.25 & 1.5 & 1.75 & 2.0
\end{array}\right] \mu \mathrm{m}
\end{gathered}
$$

The plots of Figure 3(b)-(e) show the results of using a given tiplength and all the depth values for the four videos used. The $\mathrm{y}$-axis is the median accuracy and the $\mathrm{x}$-axis is the tolerated percent error between the automatic and manual measurements (the threshold used to classify an 
automatic measurement as correct or incorrect). According to these plots, the most accurate depthvalues are $1.75 \mu \mathrm{m}$ and $2.0 \mu \mathrm{m}$ regardless of the tiplength. However, the highest accuracies are achieved when Tiplength $=20 \mu \mathrm{m}$ and depth $=[1.75$ or 2$] \mu \mathrm{m}$. Incidentally, these measurement parameters define a region that is similar in location and size to the manually drawn measurement region.

\section{User-interface of our tool TipQAD}

Figure 4 shows a snapshot of the TipQAD user interface. The interface is grouped into 3 major regions: segmentation parameters, measurement parameters, and analysis. At the start of the program, the user chooses the type of source files (video, folder, or .lif file). The default

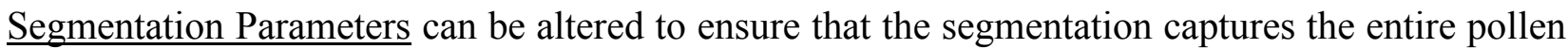
tube. The default Measurement Parameters can also be changed and the measurement regions visualized to ensure proper measurements. Lastly, the Analysis parameters are set and the program analyzes the specified frames. At the end of the analysis, the user can visualize measurement regions and the associated tip membrane distribution by selecting a frame index from the "Display Regions on Image" menu. The accumulated data can be saved by clicking on "Save Data". Table 1 lists the input parameters for TipQAD as well as a short description of each parameter and its default value. At the start of the program, the user can alter the values of these parameters.

Table 1: Table describing user inputs to TipQAD, their units and default values.

\begin{tabular}{|l|l|c|}
\hline Input & Description [units] & Default \\
\hline$R_{\text {LoG }}$ & Radius of the LoG filter [pixels] & 17 \\
\hline$R_{\text {Entropy }}$ & Radius of the entropy filter [pixels] & 9 \\
\hline Smooth contour & $($ on $=$ yes, off $=$ no) Smooth segmented contour & on \\
\hline Resize images & $($ on $=$ yes, off $=$ no) Resize the image to $[256 \times 256]$ & on \\
\hline Winsize & Width of fluorescence search space $[\mu m]$ & 5 \\
\hline Pixel scale & Size of a pixel $[\mu m]$ & 0.0758 \\
\hline Arclength & Half the length of membrane measurement line $[\mu m]$ & 10 \\
\hline Tiplength & Half the length of cytoplasm measurement area $[\mu \mathrm{m}]$ & 10 \\
\hline
\end{tabular}




\begin{tabular}{|l|l|c|}
\hline Depth & Distance from contour into the cytoplasm $[\mu \mathrm{m}]$ & 2 \\
\hline Mactime & Microscope acquisition time for a single image [ $\mathrm{sec}]$ & 1 \\
\hline Delay & Microscope delay time between two images [ $\mathrm{sec}]$ & 1 \\
\hline First frame index & Index of the first frame to be analyzed & 1 \\
\hline Spacing & Spacing between analyzed images & Video length \\
\hline Last frame index & Index of the last frame to be analyzed & on \\
\hline Display results & (on = yes, off = no) Display dynamics during execution & \\
\hline
\end{tabular}
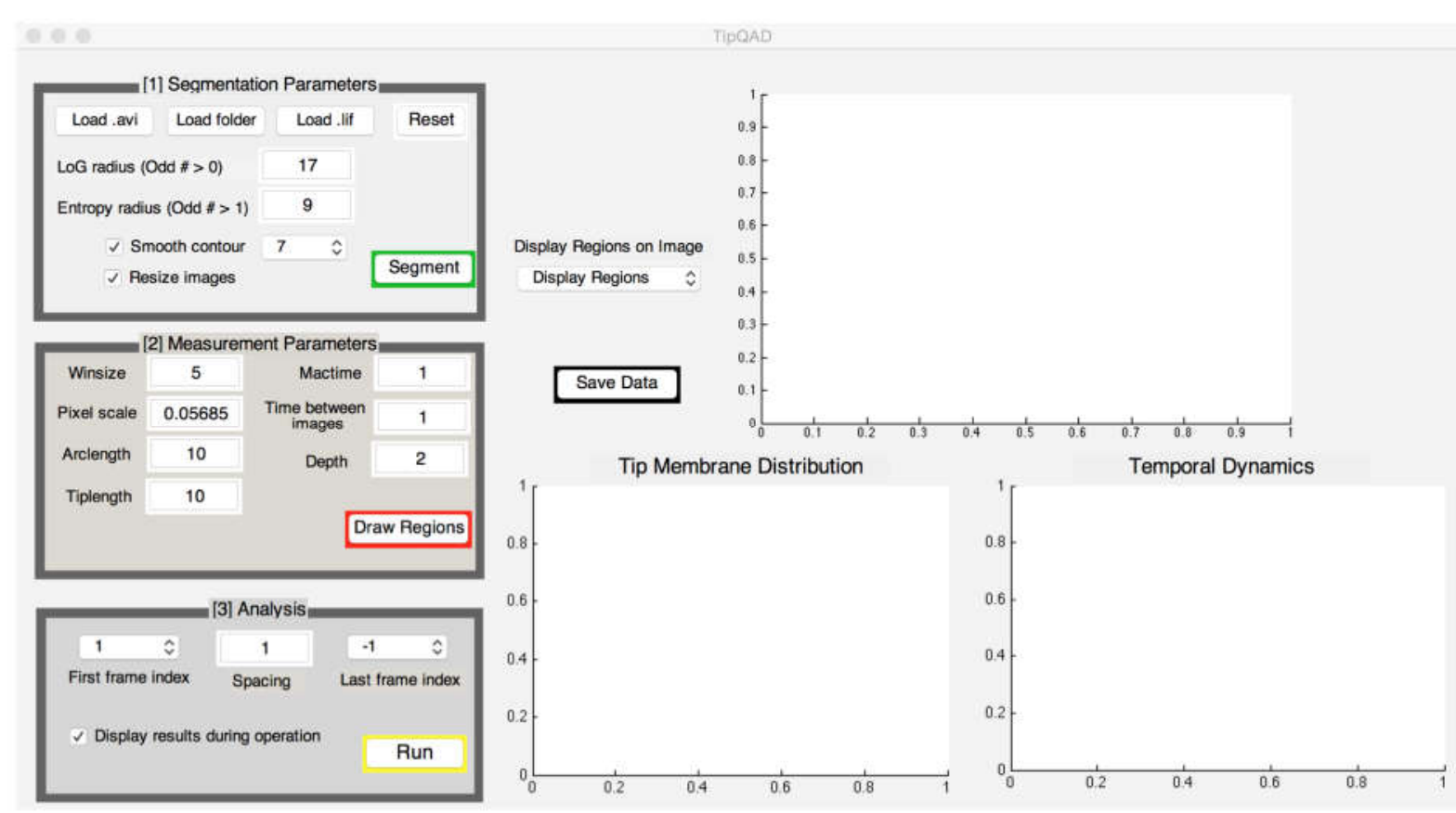

Figure 4: Display of cell analysis during program execution. TipQAD interface where the user selects what type of sequence to analyze, set/check segmentation parameters, set/check the measurement regions on the image, and set the range of images to be analyzed. As the analysis proceeds, the top image displays the previous image and measurement regions, the bottom left graph shows the fluorescence distribution along the tip membrane and the right graph shows the temporal average of both membrane (blue:solid) and tip cytoplasm (orange:dashed) regions. The "Display Regions on image" options is used after the analysis to visualize the measurement regions on any of the analyzed images. "Save Data" saves the analyzed data. By adjusting the First/Spacing/Last indices, the user can analyze (and save) selected portions of the dataset. 


\section{Making measurements}

In this section, we compare manual measurements for cell length, the intensity of PM protein fluorescence and actin filaments to those made by TipQAD. Manual measurements are obtained by trained users using Image $\mathrm{J}^{20}$ software. The accuracy of TipQAD is determined by the following equation:

$$
\text { Accuracy }=\frac{\sum\left(\frac{\left|C_{\text {auto }}-C_{\text {manual }}\right|}{C_{\text {manual }}} \leq \epsilon\right)}{\text { length }\left(C_{\text {manual }}\right)}
$$

where $|\cdot|$ is the absolute value operator, $\epsilon \in[01]$ is the acceptance threshold $(0=0 \%, 1=$ $100 \%), C_{(\cdot)}$ denotes the measurements obtained using TipQAD ( $\left.\mathrm{C}_{\text {auto }}\right)$ and manual measurements $\left(\mathrm{C}_{\text {manual }}\right)$. The numerator determines the number of measurement points that are within $\epsilon$ of the manual value, i.e. if an automatically measured value falls within $\epsilon-\%$ of the manual value, then it is considered a correct measurement. For example, 90 is within $30 \%$ of 70 because $\left(\frac{|90-70|}{70}\right)=$ $0.2857<0.3$.

\section{Cell Length vs. Time}

The length of the tip-growing cell over time is determined by an analysis of the collected vertex locations. These locations form a trajectory of the tip in a video. To minimize fluctuations in this trajectory, we smooth the trajectory using a moving-average process. The smoothing process continues until the rate of change between two iterations, measured by the gradient of the meansquared-error, falls below a specified threshold $\left(1 \times 10^{-4}\right)$. The smoothed curve at iteration $(t+1)$ is the result of a convolution operation between the curve at iteration $(t)$ with a smoothing function. The Euclidean distance between the smoothed vertices is computed and summed to give the length of the cell over time. The cell length in the first frame is considered to be 0 . Figure 5 shows length measurements and accuracy statistics for 5 videos of in vitro-growing Arabidopsis pollen tubes expressing CRIB4-GFP. The plot in Figure 5B shows that the accuracy of automated measurements increases as more error is accepted by the user. If the user accepts a $10 \%$ error, then on average, TipQAD is $75.5 \%$ close to the manual values. If the user accepts a $20 \%$ error, then on average 
TipQAD is $81.5 \%$ close to the manual values. The shaded region around the median line indicates the $1^{\text {st }}$ and $3^{\text {rd }}$ quartiles at each error threshold.
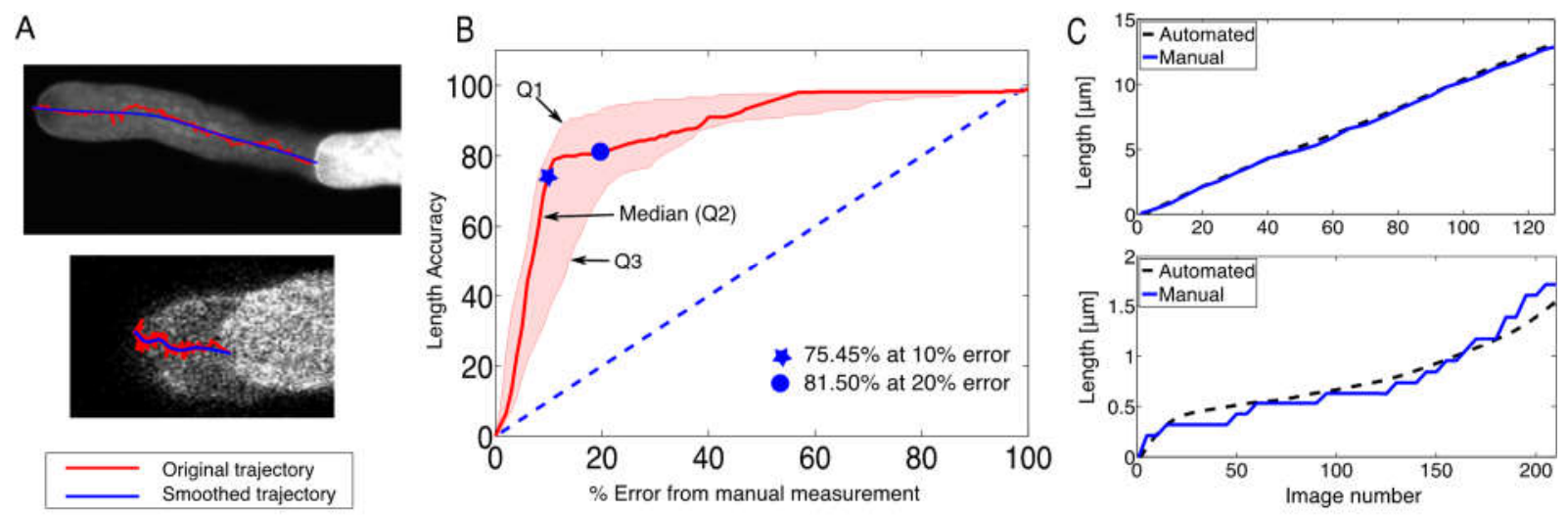

Figure 5: Comparing manual vs. automated measurements for cell length over time for 5 experimental videos. (A) Overlay of the trajectory of vertices on the start/end images from two videos. (B) Median value of accuracy, bounded by first and third quartile values, of automated length vs. error from manual length. (C) Examples of automated length measurements (dashed) vs. manual length measurements (solid) for two videos.

\section{Fluorescence Measurements on the PM of the Tip}

In these experiments, we used GFP-tagged PRK1 (AT5G35390), which is a receptor-like kinase in Arabidopsis and localizes to the PM at the tip of pollen tubes, as a membrane marker protein. The model input parameters are at their default values except for the arclength whose value is set to $6 \mu \mathrm{m}$. Membrane fluorescence measurements are made along the tip region. The desired measurement distance is specified by the user-defined variable arclength, which specifies a maximum distance on either side of the vertex along the PM. Contour (PM) points that are within the arclength-distance of the vertex are used for measurements. Since the contour is based on automated segmentation, there is no guarantee that it falls precisely at the optimal location (as drawn by a user). To alleviate this problem, we specify a distance of winsize-pixels from cell contour into the cytoplasm. The optimal measurement location for membrane dynamics is found in this region using seam carving ${ }^{21,22}$. Seam carving is a process of finding the minimum (or maximum) path of resistance, or total energy (seam) when traversing a region from one end to the other. For our purposes, seam carving finds the path of maximum fluorescence from one end of the tip measurement line to the other. Figure 6 shows a comparison of measurements made using ImageJ and the proposed method. 

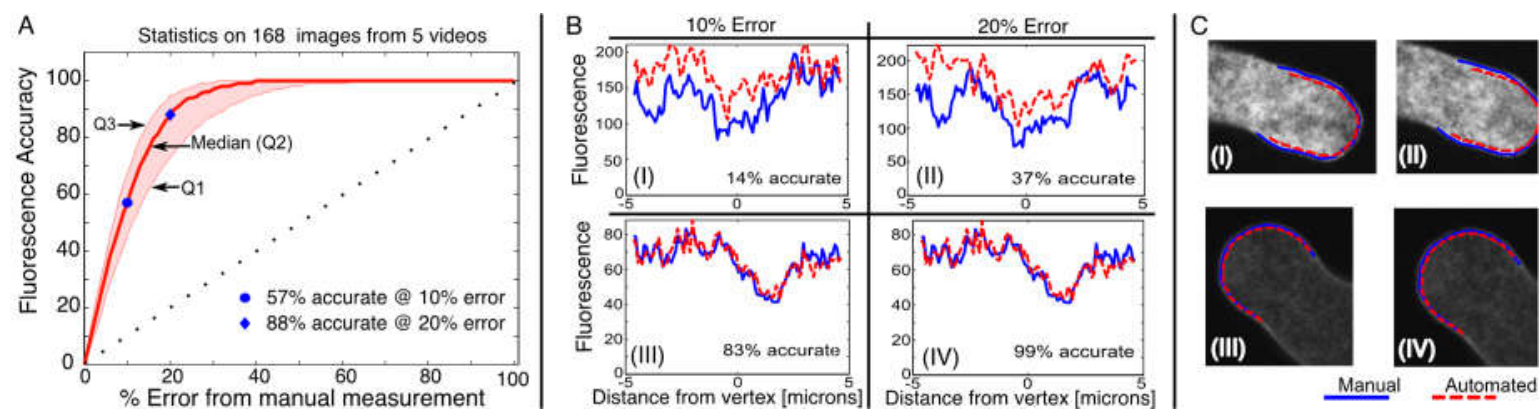

Figure 6: Comparing fluorescence measurements from user-drawn vs. model-detected regions. (A) Median fluorescence accuracy (bounded by first and third quartile statistics) for comparing automated measurements to manual measurements for 5 videos. (B) Sample tip fluorescence plots from manual-drawn (blue:solid) vs. model-detected (red:dashed) regions. At a threshold of $10 \%$ error, $\mathrm{B}(\mathrm{I})$ shows the most error and $\mathrm{B}(\mathrm{III})$ the least error. At a threshold of $20 \%$ error, B(II) shows the most error and B(IV) the least error. (C) Images showing manual and automated tip measurement lines. For each image, fluorescence values along measurement lines are shown in the corresponding image in (B).

\section{Fluorescence Measurements in the Tip Cytoplasm}

Another important measurement in the study of tip growth is the distribution of cytoplasmic molecules or structures such as actin filaments, ions, and vesicles in the cell tip ${ }^{23}$. Figure 7A shows the selected regions for both manual and automated measurements using Lifeact-EGFP as a marker for actin filaments. It has been shown that both Arabidopsis and tobacco pollen tubes have a population of fine actin filaments in the cytoplasm close to the PM of the extreme tip ${ }^{24}$. The width and depth of the cytoplasm measurement region are user-defined parameters where the width $=$ $2 \times$ tiplength. In this region, we record the average fluorescence value and compare it with the results obtained by the automated process. Figure $8 \mathrm{~B}$ shows the accuracy of automated measurements compared to the manual measurements. The x-axis indicates a measure of how far an automated measurement point is from the corresponding manual measurement (equation (4)). The y-axis shows the median accuracy value bounded by the first (lower) and third (upper) quartile statistics. This figure indicates that the automated measurements are close to the manual measurements. 

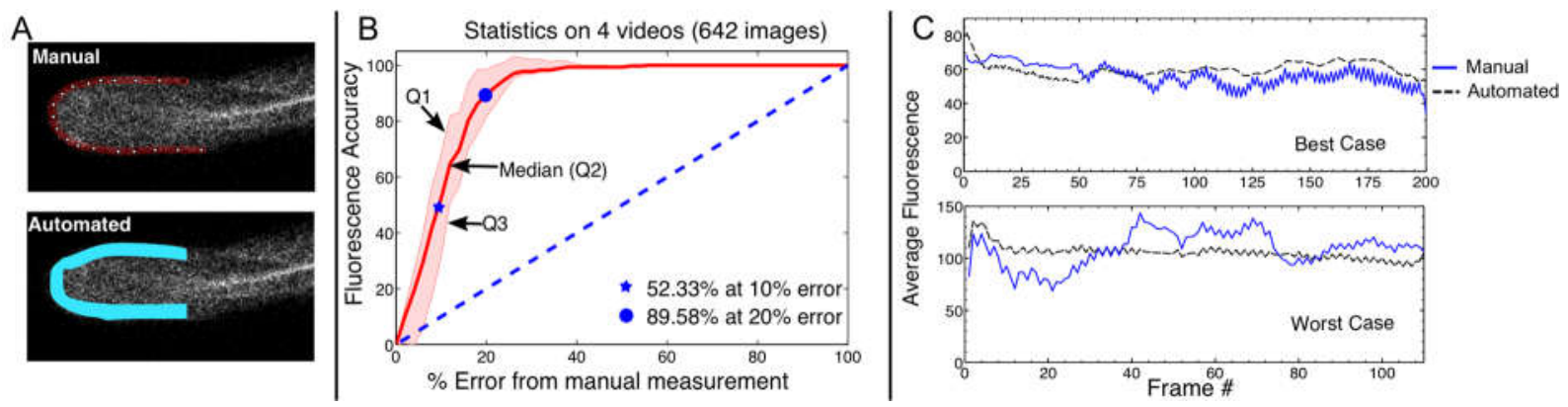

Figure 7: Comparing manual vs. automated tip cytoplasm measurements. (A) Sample images showing manual and automated measurement regions. (B) Plot of the median fluorescence accuracy bounded by first and third quartile statistics of the automated method. (C) Plots of manual and automated measurements of average fluorescence for 2 videos.

\section{Measurements on Root Hairs}

As mentioned above, TipQAD can be used to analyze other tip-growing cells. Therefore, we took a previously published video of a growing root hair cell expressing YFP-RABA $4 b^{25}$ for analyzing using our tool, TipQAD. Figure 8 shows some sample frames from the above analysis. The detection and measurement processes are the same as described in the preceding sections. The first image shows the measurement regions overlaid on the first frame of the sequence. Subsequent images show selected frames and their associated measurement regions. In this example, the segmentation boundary is not as accurate as desired (e.g. on the fourth image). These inaccuracies are largely due to intensity variations in the image over time. However, TipQAD is able to obtain an estimate of the measurement regions relatively close to the expected locations. 

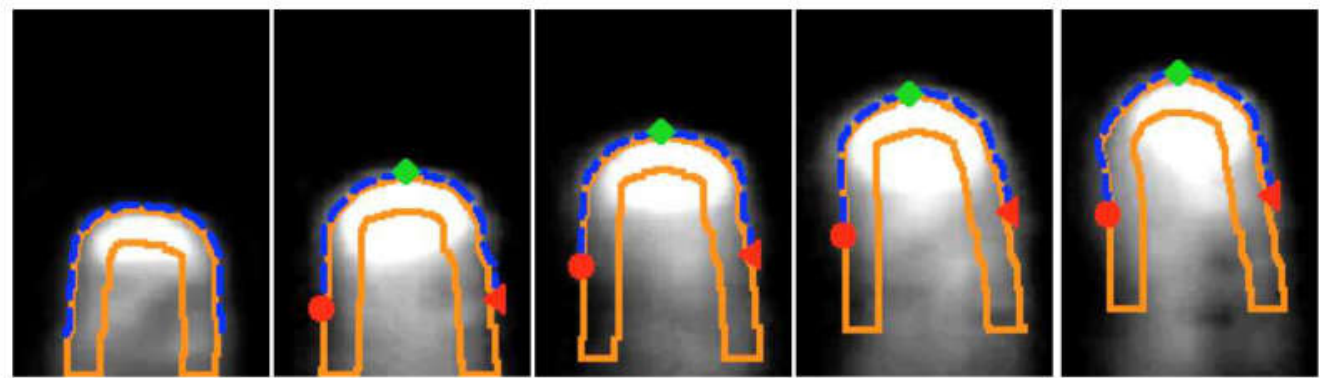

Measurement

Regions

Time

Figure 8: Sample frames from the analysis of a video of a growing root hair cell. The image on the left shows the initial measurement regions on the first frame of the video sequence. The subsequent images show the root hair at various growth stages with the associated measurement regions.

\section{Application of TipQAD to FRAP (Fluorescence Recovery After Photobleaching) Analysis in Tip-growing Cells}

TipQAD also has the potential to aid in analyzing FRAP experiments at the apical plasma membrane and in the cytoplasm. Figure 9 shows temporal measurements of fluorescence intensity in the tip region during a FRAP experiment, together with sample images at various time points. The video contains 24 frames of a FRAP experiment on a pollen tube expressing PRK1-GFP, where the PRK1-GFP on the tip PM was photobleached. The images are of size 512x512 and each pixel is of size $0.0758 \mu \mathrm{m} /$ pixel. The parameters arclength and tiplength are both set to $6 \mu \mathrm{m}$. All other parameters are at their default values. The intensity in the membrane region is always higher than in the cytoplasm region. This is expected because the membrane measurement line follows the path of the highest intensity in the membrane region. Both measurement regions show a recovery of the fluorescence signal over time and a reduction towards the end of the sequence. 


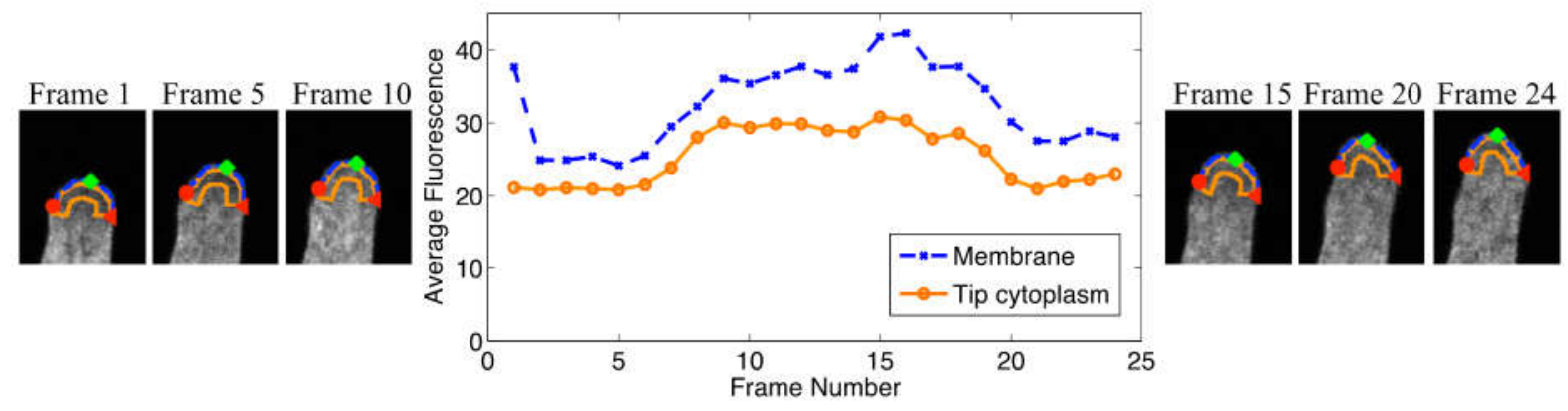

Figure 9: Plot of average membrane and cytoplasm fluorescence signals for a video sequence of a FRAP experiment on a pollen tube expressing PRK1-GFP, and some sample frames showing the measurement regions. The plots show a recovery of fluorescence in the tip region after photobleaching. The parameters arclength and tiplength are both set to $6 \mu \mathrm{m}$. All other parameters are at their default values.

\section{Discussion}

TipQAD is a tool for quantifying spatiotemporal dynamics of molecules and structures in the apical region of tip-growing cells. The effectiveness of detecting the tip region relies on the cylindrical geometry of these cells. TipQAD allows automated analysis of video images in a given region of these growing cylindrical cells, which would otherwise be conducted in a manual manner using other software packages such as ImageJ. The novelty of TipQAD lies in the application of computer vision techniques to the quantitative analysis of dynamic molecular and structural behaviors in living cells. The cell segmentation process used in TipQAD relies heavily on the edge detection of fluorescence images throughout the growing region of the cell. If the fluorescent signal is only presented in the tip region, the detection process is likely to fail. However, this dependence can be overcome by implementing a segmentation method that fuses both fluorescence and bright field image modalities ${ }^{22}$.

TipQAD greatly reduces the time for analyzing and quantifying molecular and cellular dynamics in tip-growing cells. For a set of 1000 images, average processing times for TipQAD vs. manual analysis are approximately 15 minutes vs. 250 minutes. Temporal constraints on detecting the tip region ensure consistency in tip localization over time. Furthermore, automated analysis eliminates problems posed by human error and subjectivity, hence, it gives unbiased results. With this tool, hours of user-assisted analysis are reduced to a fraction of that time. This will enable 
biologists to quickly analyze experimental videos and identify trends in fluorescence signals from large amounts of video data.

We also demonstrated that TipQAD is able to analyze the FRAP experiment. Since the basis of FRAP and FRET data analysis is the measurement of PM or cytoplasmic fluorescence signal, our method can be directly applied in FRET experiments as well. In FRAP, the fluorescence intensity at the photobleached membrane regions can be readily measured with our method after adjusting the value of arclength, and the data can be used to calculate the recovery rate. In FRET, if the protein-of-interest is localized on the cell membrane or the apical region of the cytoplasm, our method can be used to measure the fluorescence intensity in the donor, the acceptor, and the FRET channel images. The data will then be processed in the standard procedure to calculate the bleedthrough ratio, the net FRET signal, and the FRET index. In both FRAP and FRET experiments, there is usually a large number of images or videos that need quantification. Our automated tool will be very useful in these applications for its high-throughput capability. 


\section{Methods}

\section{Plant Materials}

The PRK1-GFP fragment was cloned from pLAT52::RLK-GFP described in Lee et al., 2008, and was inserted into a binary vector, pCL, which was constructed by inserting the pollen tube specific promoter LAT52 into pCAMBIA1300 using SalI and XbaI restriction sites. The resulting vector pCL::PRK1-GFP was introduced to wild-type Arabidopsis thaliana Col-0 plants using Agrobacterium-mediated floral-dip method.

The Arabidopsis plant expressing Lifeact-EGFP (pCAMBIA1300-Lat52-Lifeact-EGFP) seeds were provided by Prof. Shanjin Huang (Chinese Academy of Sciences, Beijing) ${ }^{26}$. The level of expression was determined using ImageJ by drawing a line of 5-pixel width and $20 \mu \mathrm{M}$ in length around the pollen tube tip and as close to the edge of the pollen tube as possible. The mean intensity of LifeACT-GFP along this line was then calculated.

\section{Plant Growth and Pollen Tube Preparation}

Transgenic Arabidopsis thaliana plants were grown at $22^{\circ} \mathrm{C}$ in a growth room under a light regime of $16 \mathrm{~h}$ of light and $8 \mathrm{~h}$ of dark. To generate pollen tubes for imaging, pollen grains were germinated at $28^{\circ} \mathrm{C}$ for $2-3$ hours on a solid pollen germination medium containing $18 \%(\mathrm{w} / \mathrm{v})$ sucrose, $0.01 \%$ (w/v) boric acid, $1 \mathrm{mM} \mathrm{CaCl}_{2}, 1 \mathrm{mM} \mathrm{Ca}\left(\mathrm{NO}_{3}\right)_{2}, 1 \mathrm{mM} \mathrm{MgSO}_{4}$, and 0.5\% (w/v) agar.

\section{Confocal Imaging}

Imaging of PRK1-GFP and Lifeact-EGFP in pollen tubes was performed on Leica SP2 and SP5 laser scanning confocal microscopes, respectively. PRK1-GFP and Lifeact-EGFP were excited with a $488 \mathrm{~nm}$ Argon laser line and detected at 498-560 nm. For the FRAP experiment on the pollen tube expressing PRK1-GFP, a $405 \mathrm{~nm}$ laser was used to photobleach the PRK1-GFP on the apical PM as described in Lee et al., 2008.

\section{Manual Image Quantification}

Manual image quantification was performed using the ImageJ software (version $1.45 \mathrm{~s}$ ). To measure the fluorescence intensity on the plasma membrane, a segmented line was drawn along the 
cell periphery at the tip region. To measure the fluorescence intensity in the cytoplasm, a polygon was drawn to cover the region of interest.

\section{References}

1. Yang, Z. Cell polarity signaling in arabidopsis. Annu. Rev. Cell Dev. Biol. 24, 551-575 (2008).

2. Dumais, J., Shaw, S. L., Steele, C. R., Long, S. R. \& Ray, P. M. An anisotropicviscoplastic model of plant cell morphogenesis by tip growth. Int. J. Dev. Biol. 50, 20922 (2006).

3. Hepler, P. K., Rounds, C. M. \& Winship, L. J. Control of cell wall extensibility during pollen tube growth. Mol. Plant 6, 998-1017 (2013).

4. Fayant, P. et al. Finite element model of polar growth in pollen tubes. Plant Cell 22, 2579-93 (2010).

5. Winship, L. J., Obermeyer, G., Geitmann, A. \& Hepler, P. K. Under pressure, cell walls set the pace. Trends Plant Sci. 15, 363-369 (2010).

6. Qin, Y.\& Yang, Z. Rapid tip growth: insights from pollen tubes. Semin. Cell Dev. Biol. 22, 816-824 (2011).

7. Cheung, A. Y. \& Wu, H. Structural and signaling networks for the polar cell growth machinery in pollen tubes. Annu. Rev. Plant Biol. 59, 547-572 (2008).

8. Chebli, Y., Kroeger, J. \& Geitmann, A. Transport logistics in pollen tubes. Mol. Plant 6, 1037-1052 (2013).

9. Moscatelli, A. et al. Distinct endocytic pathways identified in tobacco pollen tubes using charged nanogold. J. Cell Sci. 120, 3804-3819 (2007).

10. Lee, Y. J., Szumlanski, A., Nielsen, E. \& Yang, Z. Rho-GTPase-dependent filamentous actin dynamics coordinate vesicle targeting and exocytosis during tip growth. J. Cell Biol. 181, 1155-1168 (2008).

11. Gu, Y. et al. A Rho family GTPase controls actin dynamics and tip growth via two counteracting downstream pathways in pollen tubes. J. Cell, Biol. 169, 127-138 (2005).

12. Li, H., Lin, Y., Heath, R. M., Zhu, M. X. \& Yang, Z. Control of pollen tube tip growth by a Rop GTPase-dependent pathway that leads to tip-localized calcium influx. Plant Cell 11, 1731-1742 (1999). 
13. Steinhorst, L. \& Kudla, J. Calcium - a central regulator of pollen germination and tube growth. Biochim. Biophys. Acta 1833, 1573-81 (2013).

14. Kroeger, J. H., Geitmann, A. \& Grant, M. Model for calcium dependent oscillatory growth in pollen tubes. J. Theor. Biol. 253, 363-374 (2008).

15. Feijó, J. A. et al. Cellular oscillations and the regulation of growth: the pollen tube paradigm. BioEssays 23, 86-94 (2001).

16. Hwang, J.-U., Gu, Y., Lee, Y.-J. \& Yang, Z. Oscillatory ROP GTPase activation leads the oscillatory polarized growth of pollen tubes. Mol. Biol. Cell 16, 5385-5399 (2005).

17. Hill, A. E., Shachar-hill, B., Skepper, J. N., Powell, J. \& Shachar-hill, Y. An osmotic model of the growing pollen tube. PLoS One 7, e36585 (2012).

18. Pilu, M., Fitzgibbon, A. W., Hill, F., Eh, E. \& Fisher, R. B. Direct least square fitting of ellipses. IEEE Trans. Pattern Anal. Mach. Intell. 21, 476-480 (1999).

19. Pilu, M., Fitzgibbon, A. W., Fisher, R. B., Hill, F. \& Eh, E. Ellipse-specific direct leastsquare fitting. in International Conference on Image Processing (1996).

20. Schneider, C. A., Rasband, W. S. \& Eliceiri, K. W. NIH Image to ImageJ: 25 years of image analysis. Nat Meth 9, 671-675 (2012).

21. Shamir, A. \& Avidan, S. Seam carving for media retargeting. Commun. ACM 3, 77-85 (2009).

22. Tambo, A. L. \& Bhanu, B. Segmentation of pollen tube growth videos using dynamic bimodal fusion and seam carving. IEEE Trans. Image Process. 25, 1994 - 2004 (2006).

23. Bove, J. et al. Magnitude and direction of vesicle dynamics in growing pollen tubes using spatiotemporal image correlation spectroscopy and fluorescence recovery after photobleaching. Plant Physiol. 147, 1646-1658 (2008).

24. Fu, Y., Wu, G. \& Yang, Z. Rop GTPase-dependent dynamics of tip-localized F-actin controls tip growth in pollen tubes. J. Cell Biol. 152, 1019-1032 (2001).

25. Preuss, M. L., Serna, J., Falbel, T. G., Bednarek, S. Y. \& Nielsen, E. The Arabidopsis Rab GTPase RabA4b Localizes to the Tips of Growing Root Hair Cells. Plant Cell 16, 1589-1603 (2004).

26. Qu, X. et al. Arabidopsis villins promote actin turnover at pollen tube tips and facilitate the construction of actin collars. Plant Cell 25, 1803-1817 (2013). 


\section{Acknowledgements}

This work is funded by NSF Grant DGE 0903667 to Dr. Bhanu as the lead PI and Tambo (as NSF IGERT Fellow), and National Institute of General Medical Sciences grant (R01GM100130) to Dr. Zhenbiao Yang.

\section{Author Contribution}

A.L.T. and B.B. designed and implemented the computational method, performed data analysis and contributed to the paper. N.L. performed data collection and contributed to the paper. D.R., C.C., and I.L. performed data collection and contributed to the paper. F.W. contributed to the computational method. Z.Y. designed the experiments, supervised the data collection and contributed to the paper. All authors participated in various discussions.

\section{Additional Information}

\section{Competing Financial Interest}

The authors declare no competing financial interest. 